\title{
A GENERAL APPROXIMATION METHOD IN THE THEORY OF PLATES OF SMALL DEFLECTION*
}

\author{
BY \\ M. ZBIGNIEW KRZYWOBLOCKI (University of Illinois)
}

Introduction. The methods developed up to now in the theory of plates do not lend themselves readily to the solution of the problem in the case of an arbitrary shape of the plate. The papers of Nadai, Navier, Reissner, Southwell, and others, deal only with plates of special shapes. In the present paper the author develops a new method which aims at the solution of the problem quite independently of the shape of the plate. The outstanding feature of this method is that it is entirely independent of the shape of the plate; its success depends, however, on the type of loading. If there is no lateral load, i. e., if the forces act in the plane of the plate, the method is successful independently of "whether the forces are distributed uniformly or not. Thus, the critical stresses of a plate of arbitrary shape can be found. If there is a lateral load, the method is not always successful. There are cases in which it is not possible to find a particular solution or a fundamental integral of a non-homogeneous linear partial differential equation of the fourth order with the aid of the methods now available in mathematics.

In general, the method involves the following two steps:

(a) to obtain a function which satisfies the linear partial differential equation of the fourth order for the deflection of the plate, but does not satisfy the boundary conditions,

(b) to obtain a solution of this partial differential equation which satisfies the boundary conditions precisely or approximately.

In the case of a linear homogeneous partial differential equation of the fourth order with constant coefficients, there exists the possibility of separating the variables, and a complete set of functions can generally be found with the aid of conventional methods. The solution then is a simple series of these functions with coefficients which are determined from the boundary conditions. In the case of a linear homogeneous partial differential equation of the fourth order with variable coefficients, the problem becomes much more complicated since, in this case, the variables cannot be separated. A method given by S. Bergman [1] $\dagger$ then leads to a complete set of functions, and in particular to convergent expansions in terms of these functions. The complete solution of the differential equation is given by a double series.

To keep the possibility of applying the present method to the case where the forces acting in the plane of the plate are not distributed uniformly, the author chose Bergman's method. In order not to obscure the whole procedure by long calculations, the forces have been assumed to be uniformly distributed in the plane of the plate in the numerical example elaborated in the present paper; i.e., the coefficients in the linear homogeneous partial differential equation of the fourth order are assumed constant.

*Received Aug. 4, 1947. These investigations were carried out when the author was a post-doctorate Fellow in the Program of Advanced Instruction and Research in Mechanics at Brown University. The author wishes to acknowledge his indebtedness to Dr. S. Bergman, Harvard University, for helpful advice and suggestions and to Professor G. H. Handelman, Brown University, for some corrections during the preparation of the manuscript.

$\dagger$ Numbers in the brackets refer to the bibliography at the end of the paper. 
However, this example illustrates the general method. In the case where the forces in the plane of the plate are not distributed uniformly, the general method remains unchanged. On the other hand, some changes have to be introduced into the numerical procedure and this has been explained throughly in Ref. 10. Thus, with a certain amount of additional numerical work, the present method may be extended to the general case of variable coefficients. The boundary conditions are only satisfied approximately with the present method.

A brief resume of Bergman's method of solving homogeneous linear partial differential equations of the fourth order is given in Sec. 1. Since this method represents the solution in the form of infinite series, which involve in practical problems many thousands of operations of a simple nature, this technique is particularly suitable for punch-card machines. Such machines were employed in obtaining the numerical results given in this paper. The functions appearing in the infinite series are defined and evaluated, and the various partial differential equations of loaded plates with small deflections are discussed in Sections 2 and 3. As an illustration of the procedure, the method has been applied in Sec. 4 to the calculation of critical stresses in a triangular plate loaded uniformly in its plane parallel to the $x$-axis. The example shows that once the scheme for the calculations has been set up, the computations are quite straightforward and simple and may be performed by an inexperienced staff. All details of the example are explained together with the method of avoiding solution of determinants of higher order. Several final remarks close the paper.

1. Solution of linear partial differential equations of the fourth order. The proof and complete explanation of this particular method of solving linear partial differential equations of the fourth order can be found in S. Bergman's paper [1]. Only a brief resume of this method will be given here. The differential equation considered is the following:

$$
U_{z z z^{*} z^{*}}+M U_{z z}+L U_{z z^{*}}+N U_{z^{*} z^{*}}+A U_{z}+D U_{z^{*}}+C U=0 .
$$

The coefficients, $M, L, \cdots C$ are assumed to be entire functions of $z$ and $z^{*}$ where, in terms of complex notation, $x+i y=z, x-i y=z^{*}$. The function $U$ is also assumed to be a function of $z$ and $z^{*}$. When $z$ and $z^{*}$ are conjugate complex numbers, $x$ and $y$ are restricted to real values. If $x$ and $y$ are allowed to take on complex values, then $z$ and $z^{*}$ will be independent complex variables. The asterisk denotes the conjugate complex number and partial derivatives are denoted by subscripts. If one sets $U=$ $u+i v$, Eq. (1) is equivalent to a system of two real fourth order partial differential equations for two real functions $u$ and $v$. If for real values of $x$ and $y$ the functions $L$ and $C$ are real and $M=N^{*}=M_{1}+i M_{2}$, and $A=D^{*}=A_{1}+i A_{2}$, then the first of these two equations contains $u$ only and has the form:

$$
\Delta \Delta u+a u_{x x}+2 b u_{x y}+c u_{y y}+d u_{x}+e u_{\nu}+f u=0,
$$

where

$$
\begin{gathered}
\Delta \Delta u=\frac{\partial^{4} u}{\partial x^{4}}+2 \frac{\partial^{4} u}{\partial x^{2} \partial y^{2}}+\frac{\partial^{4} u}{\partial y^{2}}, \\
a=4 L+8 M_{1}, \quad b=8 M_{2}, \quad c=4 L-8 M_{1}, \\
d=16 A_{1}, \quad \quad \quad e=16 A_{2}, \quad f=16 C .
\end{gathered}
$$


In applications, we are mostly interested in real equations of the form (2). However, it is simpler to work with the general equation (1) without making any special assumptions about the coefficients. The derivatives employed are the following:

$$
\begin{gathered}
U=u+i v, \\
U_{z}=\frac{\partial U}{\partial z}=\frac{1}{2}\left(\frac{\partial U}{\partial x}-i \frac{\partial U}{\partial y}\right), \quad U_{* *}=\frac{\partial U}{\partial z^{*}}=\frac{1}{2}\left(\frac{\partial U}{\partial x}+i \frac{\partial U}{\partial y}\right), \\
U_{z z^{*}}=\frac{\partial}{\partial z^{*}}\left[\frac{1}{2}\left(\frac{\partial U}{\partial x}-i \frac{\partial U}{\partial y}\right)\right]=\frac{1}{4}\left[\frac{\partial}{\partial x}\left(\frac{\partial U}{\partial x}-i \frac{\partial U}{\partial y}\right)+i \frac{\partial}{\partial y}\left(\frac{\partial U}{\partial x}-i \frac{\partial U}{\partial y}\right)\right] \\
\cdot \quad=\frac{1}{4}\left(\frac{\partial^{2} U}{\partial x^{2}}+\frac{\partial^{2} U}{\partial y^{2}}\right), \\
U_{z z}=\frac{1}{4}\left(\frac{\partial^{2} U}{\partial x^{2}}-2 i \frac{\partial^{2} U}{\partial x \partial y}-\frac{\partial^{2} U}{\partial y^{2}}\right), \\
U_{z^{*} z^{*}}=\frac{1}{4}\left(\frac{\partial^{2} U}{\partial x^{2}}+2 i \frac{\partial^{2} U}{\partial x \partial y}-\frac{\partial^{2} U}{\partial y^{2}}\right), \\
U_{z z z^{* *}}=\frac{1}{16}\left(\frac{\partial^{4} U}{\partial x^{4}}+2 \frac{\partial^{4} U}{\partial x^{2} \partial y^{2}}+\frac{\partial^{4} U}{\partial y^{4}}\right) .
\end{gathered}
$$

S. Bergman [1] has proved the following theorem concerning the solution of Eq. (1) [see Theorem 2.1. and Remark 9.1. of his paper].

Theorem. There exist four functions $E^{(k, \kappa)}\left(z, z^{*}, t\right), k=I, I I, \kappa=1,2$, which are defined for sufficiently small values, say $|z|<\rho_{1},\left|z^{*}\right|<\rho_{2}$, and for $|t| \leq 1$, possessing the following property: If $f_{\kappa}(\zeta)$ and $g_{k}(\zeta), \kappa=1,2$, are any analytic functions of $\zeta$ defined in the neighborhood of the origin, then

$$
\begin{aligned}
U\left(z, z^{*}\right)=\sum_{\kappa=1}^{2} \int_{-1}^{+1}\left\{E^{(I \kappa)}\left(z, z^{*}, t\right) f_{\kappa}\right. & {\left[\frac{1}{2} z\left(1-t^{2}\right)\right] } \\
+ & \left.E^{(I I \kappa)}\left(z, z^{*}, t\right) g_{\kappa}\left[\frac{1}{2} z^{*}\left(1-t^{2}\right)\right]\right\}\left(1-t^{2}\right)^{-1 / 2} d t,
\end{aligned}
$$

is a solution of (1). Conversely, if $U\left(z, z^{*}\right)$ is a solution of (1) defined in the neighborhood of the origin $z=z^{*}=0$, then $U$ can be represented in the form (5) by means of suitably chosen functions $f_{k}$ and $g_{k}, \kappa=1,2$.

This theorem yields a local result; i.e., this theorem states that representation (5) for $U\left(z, z^{*}\right)$ is valid only in a sufficiently small neighborhood of the origin. By proving a theorem concerning analytical continuation in the complex domain of solutions of the differential equation (1) with analytic coefficients, Bergman has shown that representation (5) is valid in the large i.e. in a finite domain. He also proved the following theorem [Theorem 11.1].

Theorem. Let $U$ be a solution of (1) represented in the form (5). Let $U$ be regular for $(x, y) \varepsilon R^{2}, E^{(k, k)}\left(z, z^{*}, t\right)$ for $|t| \leq 1,(x, y) \varepsilon R^{*^{2}}+\frac{1}{2} R^{*^{2}}$. Then the functions $f_{1}, f_{2}$, $g_{1}, g_{2}$ are regular in $\frac{1}{2} R^{2}$. By $\frac{1}{2} R^{2}$ we denote the domain obtained from domain $R^{2}$ by the transformation $z^{\mathbf{\Lambda}}=\frac{1}{2} z$. 
Because of the condition $|t| \leq 1$ in (5) this domain cannot be greater than the unit circle. In order to obtain rapid convergence of the series, the radius of the circle should not be greater than 0.5 to 0.6 for practical purposes. The functions $E^{(k, \kappa)}\left(z, z^{*}, t\right)$ introduced above have the property that

$$
\begin{aligned}
& E^{(I 1)}(z, 0, t)=E^{(I I 1)}\left(0, z^{*}, t\right)=1, \\
& E^{(I 2)}(z, 0, t)=E^{(I I 2)}\left(0, z^{*}, t\right)=0, \\
& E_{z^{*}}^{(I)}(z, 0, t)=E_{z}^{(I I 1)}\left(0, z^{*}, t\right)=0, \\
& E_{z^{*}}^{(I 2)}(z, 0, t)=E_{z}^{(I I 2)}\left(0, z^{*}, t\right)=1 .
\end{aligned}
$$

Each of the functions $E\left(z, z^{*}, t\right)$ can be represented in the form

$$
E\left(z, z^{*}, t\right)=P^{(0)}\left(z, z^{*}\right)+\sum_{v=1}^{\infty} t^{2 v} z^{v} P^{(v)}\left(z, z^{*}\right),
$$

where the functions $P^{(v)}$ are to be determined. They are defined by the following system of differential equations, which arise from the application of the linear differential operator $L_{\mathbf{1}}^{\mathbf{A}}(E)$ and from the solution of the equation $L_{\mathbf{1}}^{\mathbf{A}}(\dot{E})=0$ :

$$
\begin{gathered}
D_{1}\left[P^{(0)}\right]=0, \\
D_{1}\left[P^{(1)}\right]=-4 D_{1}\left[P_{3}^{(0)}\right]-2 D_{2}\left[P^{(0)}\right], \\
D_{1}\left[P^{(n+2)}\right]=-\frac{1}{n^{2}+2 n+3 / 4}\left[D_{1}\left[P_{3 z}^{(n)}\right]+(2 n+1) D_{1}\left[P_{s}^{(n+1)}\right]\right. \\
+D_{2}\left[P_{s}^{(n)}\right]+\left(n+\frac{1}{2}\right) D_{2}\left[P^{(n+1)}\right] \\
\left.+N P_{z^{*} z^{*}}^{(n)}+D P_{z^{*}}^{(n)}+C P^{(n)}\right], \quad(n=0,1,2 \cdots),
\end{gathered}
$$

where

$$
D_{1}(H)=H_{z^{*} z^{*}}+M H \text { and } D_{2}(H)=L H_{z^{*}}+A H \text {. }
$$

System (8) is a system of differential recurrence formulas for the functions $P^{(n)}$. After $P^{(0)}, P^{(1)}, \ldots P^{(n+1)}$ have been determined, $P^{(n+2)}$ can be obtained by solving the ordinary differential equation, where only $P^{(n)}$ and $P^{(n+1)}$ appear. There exist two sequences of functions $P^{(11 n)}\left(z, z^{*}\right)$ and $P^{(12 n)}\left(z, z^{*}\right), n=0,1,2 \cdots$, satisfying the differential equations (8), $n=0,1,2 \cdots$, and such that

$$
\begin{array}{ll}
P^{(I 10)}(z, 0)=1, & P_{z^{*}}^{(110)}(z, 0)=0, \\
P^{(I 1 n)}(z, 0)=0, & P_{z^{*}}^{(I 1 n)}(z, 0)=0, \quad(n=1,2 \cdots),
\end{array}
$$

and

$$
\begin{array}{ll}
P^{(120)}(z, 0)=0, & P_{z^{*}}^{(2 *)}(z, 0)=1, \\
P^{(12 n)}(z, 0)=0, & P_{z^{*}}^{(12 n)}(z, 0)=0, \quad(n=1,2 \cdots) .
\end{array}
$$


The conditions and the proof of uniform and absolute convergence of these series have been given by Bergman [1]. Two other series for $P^{(v)}$ are obtained by interchanging $z$ and $z^{*}$, by interchanging differentiation with respect to $z$ and $z^{*}$ everywhere and by replacing $M$ and $A$ by $N$ and $D$ respectively, and conversely.

Let us take into consideration only one function $E$. Substituting Eq. (7) into (5), one obtains:

$$
\begin{aligned}
U\left(z, z^{*}\right)=\int_{-1}^{+1} & P^{(0)}\left(z, z^{*}\right) f\left[\frac{z\left(1-t^{2}\right)}{2}\right]\left(1-t^{2}\right)^{-1 / 2} d t \\
& +\int_{-1}^{+1} t^{2} z P^{(1)}\left(z, z^{*}\right) f\left[\frac{z\left(1-t^{2}\right)}{2}\right]\left(1-t^{2}\right)^{-1 / 2} d t \\
& +\int_{-1}^{+1} t^{4} z^{2} P^{(2)}\left(z, z^{*}\right) f\left[\frac{z\left(1-t^{2}\right)}{2}\right]\left(1-t^{2}\right)^{-1 / 2} d t+\cdots
\end{aligned}
$$

Let us assume that the integral

$$
I_{1}=\int_{-1}^{+1} f\left[\frac{z\left(1-t^{2}\right)}{2}\right]\left(1-t^{2}\right)^{-1 / 2} d t
$$

may be expanded into a simple power series of the form

$$
I_{1}=\int_{-1}^{+1} f\left[\frac{z\left(1-t^{2}\right)}{2}\right]\left(1-t^{2}\right)^{-1 / 2} d t=\sum_{v=0}^{\infty} z^{\nu} .
$$

To this end let us assume that the function $f(z, t)$ under the integral sign can be expanded into a power series of the form

$$
f\left[\frac{z\left(1-t^{2}\right)}{2}\right]=\sum_{p=0}^{\infty} c_{\nu} \frac{z^{\prime}\left(1-t^{2}\right)^{\prime}}{2^{\prime}},
$$

This series (11b) can then be inserted in Eq. (11a), giving:

$$
\begin{aligned}
I_{1}=\int_{-1}^{+1} f\left[\frac{z\left(1-t^{2}\right)}{2}\right]\left(1-t^{2}\right)^{-1 / 2} d t & =\int_{-1}^{+1} \sum_{\nu=0}^{\infty} c_{\nu}\left[\frac{z^{y}\left(1-t^{2}\right)^{\nu}}{2^{\gamma}}\right]\left(1-t^{2}\right)^{-1 / 2} d t \\
& =\sum_{\nu=0}^{\infty} z^{\nu}
\end{aligned}
$$

If we make the change of variable $t^{2}=p$, then

$$
d t=\frac{d p}{2(p)^{1 / 2}}
$$

and

$$
\int_{-1}^{+1}\left(1-t^{2}\right)^{\nu-1 / 2} d t=\int_{0}^{1}(1-p)^{\nu-1 / 2} p^{-1 / 2} d p=\frac{\Gamma(\nu+1 / 2) \Gamma(1 / 2)}{\Gamma(\nu+1)} .
$$

Equation (14) can also be written in terms of a Beta-function; namely,

$$
\frac{\Gamma(\nu+1 / 2) \Gamma(1 / 2)}{\Gamma(\nu+1)}=B\left(\nu+\frac{1}{2}, \frac{1}{2}\right)
$$


since $B(p, q)=[\Gamma(p) \Gamma(q)] /[\Gamma(p+q)]$. Thus:

$$
I_{1}=\sum_{\nu=0}^{\infty} c_{\nu} \frac{1}{2^{\nu}} \frac{\Gamma(\nu+1 / 2) \Gamma(1 / 2)}{\Gamma(\nu+1)} z^{\nu}=\sum_{\nu=0}^{\infty} z^{\nu}
$$

From Eq. (14b) we find:

$$
c_{\nu}=2^{\nu} \frac{\Gamma(\nu+1)}{\Gamma(\nu+1 / 2) \Gamma(1 / 2)} .
$$

In the first integral $I_{1}$ of Eq. (11) the simple form of the power series $\left(I_{1}=\sum_{\nu=0}^{\infty} z^{\prime}\right)$ will be used, whereas the form (11b) together with the values of the coefficient, found from Eq. (15), will be used in the second, third, etc. terms in the series (11). For the second term in the series (11), we must consider an expression of the form:

$$
I_{2}=\int_{-1}^{+1} t^{2} z f\left[\frac{z\left(1-t^{2}\right)}{2}\right]\left(1-t^{2}\right)^{-1 / 2} d t .
$$

Substitute in Eq. (16) for $f(z, t)$ the series (11b) together with the values of the coefficients, calculated from Eq. (15):

$$
\begin{aligned}
I_{2} & =\int_{-1}^{+1} t^{2} z \sum_{\nu=0}^{\infty} c_{\nu} \frac{z^{\nu}\left(1-t^{2}\right)^{\nu}}{2^{\nu}}\left(1-t^{2}\right)^{-1 / 2} d t \\
& =\sum_{\nu=0}^{\infty} \frac{\Gamma(\nu+1)}{\Gamma(\nu+1 / 2) \Gamma(1 / 2)} z^{\nu+1} \cdot \int_{-1}^{+1} t^{2}\left(1-t^{2}\right)^{\nu-1 / 2} d t \\
& =\sum_{\nu=0}^{\infty} \frac{\Gamma(\nu+1)}{\Gamma(\nu+1 / 2) \Gamma(1 / 2)} z^{\nu+1} \frac{\Gamma(\nu+1 / 2) \Gamma(3 / 2)}{\Gamma(\nu+2)} \\
& =\sum_{\nu=0}^{\infty} \frac{\Gamma(3) \cdot}{2^{2} \Gamma(2)} \frac{z^{\nu+1}}{(\nu+1)}=\sum_{\nu=0}^{\infty} \frac{1}{2} \frac{z^{\nu+1}}{(\nu+1)} .
\end{aligned}
$$

The third term can be evaluated in a similar manner. We easily obtain:

$$
\begin{aligned}
\int_{-1}^{+1} t^{4} z^{2} f\left[\frac{z\left(1-t^{2}\right)}{2}\right]\left(1-t^{2}\right)^{-1 / 2} d t & =\frac{\Gamma(\nu+1)}{\Gamma(\nu+1 / 2) \Gamma(1 / 2)} z^{\nu+2} \frac{\Gamma(\nu+1 / 2) \Gamma(5 / 2)}{\Gamma(\nu+3)} \\
= & \frac{\Gamma(5)}{2^{4} \Gamma(3)} \frac{z^{\nu+2}}{(\nu+1)(\nu+2)}=\frac{3}{4} \frac{z^{\nu+2}}{(\nu+1)(\nu+2)}, \quad \text { etc. }
\end{aligned}
$$

Of course, the functions $P^{(n)}$ will be functions of $z^{*}$ alone, if expressions (12) to ${ }^{\prime}(17)$ are functions of $z$ alone and vice versa. Taking all four sequences into account, we find:

$$
\begin{aligned}
U\left(z, z^{*}\right)=\sum_{n=0}^{\infty} & \sum_{\nu=0}^{\infty} K_{n \nu} P^{(I 1 n)}\left(z^{*}\right) z^{n+\nu} \\
& +\sum_{n=0}^{\infty} \sum_{\nu=0}^{\infty} K_{n \nu} P^{(I 2 n)}\left(z^{*}\right) z^{n+\nu} \\
& +\sum_{n=0}^{\infty} \sum_{\nu=0}^{\infty} K_{n \nu} P^{(I I 1 n)}(z) z^{* n+\nu} \\
& +\sum_{n=0}^{\infty} \sum_{\nu=0}^{\infty} K_{n \nu} P^{(I I 2 n)}(z) z^{* n+\nu}
\end{aligned}
$$


where

$$
\begin{array}{rlrl}
K_{0 \nu}=1, & K_{10}=\frac{1}{2} \cdot \frac{1}{1}, & K_{20}=\frac{3}{4} \cdot \frac{1}{1 \cdot 2}, \quad \cdots, \\
K_{11}=\frac{1}{2} \cdot \frac{1}{2}, & K_{21}=\frac{3}{4} \cdot \frac{1}{2 \cdot 3}, \quad \cdots, \\
K_{12}=\frac{1}{2} \cdot \frac{1}{3}, & K_{22}=\frac{3}{4} \cdot \frac{1}{3 \cdot 4}, \quad \cdots, \\
\ldots \ldots \cdots, & \ldots \ldots, \cdots, \quad \cdots, \\
K_{1 \nu}=\frac{1}{2} \frac{1}{(\nu+1)}, & K_{2 \nu}=\frac{3}{4} \frac{1}{(\nu+1)(\nu+2)} \cdots
\end{array}
$$

Certain constant coefficients have to be introduced in the process of integration; these should be defined by the boundary conditions. Separation of the real and imaginary parts leads to the following form of the general solution of the partial differential equation considered:

$$
\begin{array}{rl}
U\left(z, z^{*}\right)=a_{\nu 1} & R e\left[\sum_{n=0}^{\infty} \sum_{\nu=0}^{\infty} K_{n v} P^{(I 1 n)}\left(z^{*}\right) z^{n+\nu}\right] \\
& +a_{\nu 2} \operatorname{Im}\left[\sum_{n=0}^{\infty} \sum_{\nu=0}^{\infty} K_{n \nu} P^{(I 1 n)}\left(z^{*}\right) z^{n+\nu}\right] \\
& +b_{\nu 1} \operatorname{Re}\left[\sum_{n=0}^{\infty} \sum_{\nu=0}^{\infty} K_{n v} P^{(I 2 n)}\left(z^{*}\right) z^{n+\nu}\right] \\
& +b_{\nu 2} \operatorname{Im}[\cdots]+c_{\nu 1} \operatorname{Re}[\cdots]+c_{\nu 2} \operatorname{Im}[\cdots] \\
& +d_{\nu 1} \operatorname{Re}\left[\sum_{n=0}^{\infty} \sum_{\nu=0}^{\infty} K_{n v} P^{(I I 2 n)}(z) z^{* n+\nu}\right] \\
& +d_{\nu 2} \operatorname{Im}\left[\sum_{n=0}^{\infty} \sum_{\nu=0}^{\infty} K_{n v} P^{(I I 2 n)}(z) z^{* n+\nu}\right] .
\end{array}
$$

In case Eq. (2) is taken as the starting point of these considerations, one does not need to take into account the conjugate sequences $P^{(I I)}$ since both $P^{(I)}$ and $P^{(I I)}$ will give the same results. Thus only the sequence $P^{(I)}$ need be calculated.

2. Functions $P^{(I)}$ and $P^{(I I)}$. Of course, there are many functions which satisfy conditions (8) and consequently represent the solution. From Eq. (9), we find that

$$
D_{1}\left[P^{(0)}\right]=P_{z^{*} z^{*}}^{(0)}+M P^{(0)}=0,
$$

from which it follows that

$$
P^{(0)}=\cos \left(M^{1 / 2} z^{*}\right) \text { or } P^{(0)}=\sin \left(M^{1 / 2} z^{*}\right),
$$

under the assumption that $M=$ constant. The general solution is

$$
P^{(I 10)}=A_{1} \cos \left(M^{1 / 2} z^{*}\right)+A_{2} \sin \left(M^{1 / 2} z^{*}\right) .
$$

From Eq. (10) one may easily find that $A_{1}=1$ and $A_{2}=0$. Thus

$$
P^{(110)}=\cos \left(M^{1 / 2} z^{*}\right),
$$


and similarly,

$$
\begin{gathered}
P^{(120)}=A_{1} \cos \left(M^{1 / 2} z^{*}\right)+A_{2} \sin \left(M^{1 / 2} z^{*}\right)=\frac{1}{M^{1 / 2}} \sin \left(M^{1 / 2} z^{*}\right), \\
D_{1}\left[P^{(1)}\right]=P_{z_{*}^{*}}^{(1)}+M P^{(1)}=-4 D_{1}\left[P_{s}^{(0)}\right]-2 D_{2}\left[P^{(0)}\right] \\
=-4\left[P_{s s^{* *}}^{(0)}+M P_{s}^{(0)}\right]-2\left[L P_{s^{*}}^{(0)}+A P^{(0)}\right] .
\end{gathered}
$$

For $P^{(110)}=\cos \left(M^{1 / 2} z^{*}\right)=P^{(0)}$ one obtains

$$
\begin{gathered}
P_{s}^{(0)}=P_{s z^{*} s^{*}}^{(0)}=0, \quad P_{s^{*}}^{(0)}=-M^{1 / 2} \sin \left(M^{1 / 2} z^{*}\right), \\
P_{s^{*} s^{*}}^{(1)}+M P^{(1)}=2 L M^{1 / 2} \sin \left(M^{1 / 2} z^{*}\right)-2 A \cos \left(M^{1 / 2} z^{*}\right) .
\end{gathered}
$$

The homogeneous equation $P_{. * 9 *}^{(1)}+M P^{(1)}=0$ has the solutions

$$
P^{(1)}=u_{1}=\cos \left(M^{1 / 2} z^{*}\right) \quad \text { and } \quad P^{(1)}=u_{2}=\frac{1}{M^{1 / 2}} \sin \left(M^{1 / 2} z^{*}\right) .
$$

Any conventional method might be used to solve the non-homogeneous equation (27) by finding the particular integral, for example, the method of variation of parameters. One may easily show, however, all the conventional methods have proved to be highly impractical and inconvenient for higher terms of the sequences $P^{(11 n)}$ and $P^{(12 n)}$. For this reason, the method given by Ince [2] will be used. This proved to be shorter beginning with the term $P^{(112)}$. As an illustration, let us apply this technique to the solution of Eq. (27). We note that the Wronskian

$$
\begin{aligned}
\Delta\left(u_{1}, u_{2}\right) & =\left|\begin{array}{cc}
u_{1} & u_{2} \\
u_{1}^{\prime} & u_{2}^{\prime}
\end{array}\right| \\
& =\left|\begin{array}{c}
\cos \left(M^{1 / 2} z^{*}\right)\left(M^{1 / 2}\right)^{-1} \sin \left(M^{1 / 2} z^{*}\right) \\
-M^{1 / 2} \sin \left(M^{1 / 2} z^{*}\right) \cos \left(M^{1 / 2} z^{*}\right)
\end{array}\right|=1 .
\end{aligned}
$$

The functions $V_{1}$ and $V_{2}$ are given by

$$
V_{1}=-\int \frac{u_{2}}{\Delta} r\left(z^{*}\right) d z^{*}, \quad V_{2}=\int \frac{u_{1}}{\Delta} r\left(z^{*}\right) d z^{*},
$$

where the expression $r\left(z^{*}\right)$ represents the right side of the non-homogeneous equation,

$$
r\left(z^{*}\right)=2 L M^{1 / 2} \sin \left(M^{1 / 2} z^{*}\right)-2 A \cos \left(M^{1 / 2} z^{*}\right) .
$$

After the necessary substitution and calculations are performed one obtains

$$
\begin{aligned}
& V_{1}=\frac{A}{M} \sin ^{2}\left(M^{1 / 2} z^{*}\right)-L z^{*}+\frac{L}{M^{1 / 2}} \cos \left(M^{1 / 2} z^{*}\right) \sin \left(M^{1 / 2} z^{*}\right)+C_{1}, \\
& V_{2}=L \sin ^{2}\left(M^{1 / 2} z^{*}\right)-A z^{*}-\frac{A}{M^{1 / 2}} \cos \left(M^{1 / 2} z^{*}\right) \sin \left(M^{1 / 2} z^{*}\right)+C_{2} .
\end{aligned}
$$


Finally the general solution is given by

$$
y=V_{1} u_{1}+V_{2} u_{2}=P^{(1)}=P^{(I 11)} .
$$

Condition (10) implies that $C_{1}=C_{2}=0$; and, finally,

$$
P^{(111)}=\frac{L}{M^{1 / 2}} \sin \left(M^{1 / 2} z^{*}\right)-L z^{*} \cos \left(M^{1 / 2} z^{*}\right)-\frac{A}{M^{1 / 2}} z^{*} \sin \left(M^{1 / 2} z^{*}\right) .
$$

One can easily show by direct substitution into Eq. (27), that Eq. (33) gives the required result. In a similar way, the function $P^{(121)}$ and all the others can be found. The table, given below, shows the results. In order to obtain the function $P^{(I I)}$ one has to replace $z^{*}$ by $z, M$ by $N, D$ by $A$ and vice versa.

$$
\begin{aligned}
P^{(I 10)}: & \cos \left(M^{1 / 2} z^{*}\right), \\
P^{(I 11)}: & \frac{L}{M^{1 / 2}} \sin \left(M^{1 / 2} z^{*}\right)-L z^{*} \cos \left(M^{1 / 2} z^{*}\right)-\frac{A}{M^{1 / 2}} z^{*} \sin \left(M^{1 / 2} z^{*}\right), \\
P^{(112)}: & \frac{A L}{3 M^{1 / 2}} z^{*^{2}} \sin \left(M^{1 / 2} z^{*}\right)+\left[\frac{L^{2}}{6}-\frac{A_{2}^{2}}{6 M}\right] z^{*^{2}} \cos \left(M^{1 / 2} z^{*}\right) \\
+ & {\left[\frac{A^{2}}{6 M M^{1 / 2}}-\frac{L^{2}}{6 M^{1 / 2}}+\frac{2 N M^{1 / 2}}{3}-\frac{2 C}{3 M^{1 / 2}}\right] z^{*} \sin \left(M^{1 / 2} z^{*}\right) } \\
+ & {\left[\frac{A L}{3 M}-\frac{2 D}{3}\right] z^{*} \cos \left(M^{1 / 2} z^{*}\right)+\frac{2 D}{3 M^{1 / 2}} \sin \left(M^{1 / 2} z^{*}\right), }
\end{aligned}
$$

$$
\begin{aligned}
& P^{(120)}: \frac{1}{M^{1 / 2}} \sin \left(M^{1 / 2} z^{*}\right) \\
& P^{(121)}: \frac{A}{M} z^{*} \cos \left(M^{1 / 2} z^{*}\right)-\frac{A}{M M^{1 / 2}} \sin \left(M^{1 / 2} z^{*}\right)-\frac{L}{M^{1 / 2}} z^{*} \sin \left(M^{1 / 2} z^{*}\right), \\
& P^{(122):}\left[\frac{L^{2}}{6 M^{1 / 2}}-\frac{A^{2}}{6 M M^{1 / 2}}\right] z^{*^{2}} \sin \left(M^{1 / 2} z^{*}\right)-\frac{A L}{3 M} z^{*^{2}} \cos \left(M^{1 / 2} z^{*}\right) \\
& +\left[\frac{A L}{3 M M^{1 / 2}}-\frac{2 D}{3 M^{1 / 2}}\right] z^{*} \sin \left(M^{1 / 2} z^{*}\right) \\
& +\left[\frac{-L^{2}}{6 M}-\frac{A^{2}}{2 M^{2}}-\frac{2 N}{3}+\frac{2 C}{3 M}\right] z^{*} \cos \left(M^{1 / 2} z^{*}\right) \\
& +\left[\frac{L^{2}}{6 M M^{1 / 2}}+\frac{A^{2}}{2 M^{2} M^{1 / 2}}+\frac{2 N}{3 M^{1 / 2}}-\frac{2 C}{3 M M^{1 / 2}}\right] \sin \left(M^{1 / 2} z^{*}\right),
\end{aligned}
$$




$$
\begin{aligned}
& P^{(I I 10)}: \cos \left(N^{1 / 2} z\right), \\
& P^{(I r 11)}: \frac{L}{N^{1 / 2}} \sin \left(N^{1 / 2} z\right)-L z \cos \left(N^{1 / 2} z\right)-\frac{D}{N^{1 / 2}} z \sin \left(N^{1 / 2} z\right), \\
& P^{(I I 12)}: \frac{D L}{3 N^{1 / 2}} z^{2} \sin \left(N^{1 / 2} z\right)+\left[\frac{L^{2}}{6}-\frac{D^{2}}{6 N}\right] z^{2} \cos \left(N^{1 / 2} z\right) \\
& +\left[\frac{D^{2}}{6 N N^{1 / 2}}-\frac{L^{2}}{6 N^{1 / 2}}+\frac{2 M N^{1 / 2}}{3}-\frac{2 C}{3 N^{1 / 2}}\right] z \sin \left(N^{1 / 2} z\right) \\
& +\left[\frac{\dot{D} L}{3 N}-\frac{2 A}{3}\right] z \cos \left(N^{1 / 2} z\right)+\frac{2 A}{3 N^{1 / 2}} \sin \left(N^{1 / 2} z\right), \\
& P^{(I I 20)}: \frac{1}{N^{1 / 2}} \sin \left(N^{1 / 2} z\right) \text {, } \\
& P^{(I I 21)}: \frac{D}{N} z \cos \left(N^{1 / 2} z\right)-\frac{D}{N N^{1 / 2}} \sin \left(N^{1 / 2} z\right)-\frac{L}{N^{1 / 2}} z \sin \left(N^{1 / 2} z\right), \\
& P^{(I I 22)}:\left[\frac{L^{2}}{6 N^{1 / 2}}-\frac{D^{2}}{6 N N^{1 / 2}}\right] z^{2} \sin \left(N^{1 / 2} z\right)-\frac{D L}{3 N} z^{2} \cos \left(N^{1 / 2} z\right) \\
& +\left[\frac{D L}{3 N N^{1 / 2}}-\frac{2 A}{3 N^{1 / 2}}\right] z \sin \left(N^{1 / 2} z\right) \\
& +\left[\frac{-L^{2}}{6 N}-\frac{D^{2}}{2 N^{2}}-\frac{2 M}{3}+\frac{2 C}{3 N}\right] z \cos \left(N^{1 / 2} z\right) \\
& +\left[\frac{L^{2}}{6 N N^{1 / 2}}+\frac{D^{2}}{2 N^{2} N^{1 / 2}}+\frac{2 M}{3 N^{1 / 2}}-\frac{2 C}{3 N N^{1 / 2}}\right] \sin \left(N^{1 / 2} z\right) .
\end{aligned}
$$

Direct substitution into Eq. (18) of the expressions for the sequences $P^{(11 n)}, P^{(12 n)}$, $P^{(I I 1 n)}, P^{(I I 2 n)}$, taken with a limited number of terms, and subsequent substitution of Eq. (18) into (1) show quite easily that all terms with the power $z^{\prime}$ or less than $z^{\nu}$, i.e. $z^{\nu-1}, z^{\nu-2}$ etc. cancel. The terms with the power $z^{\nu+1}$ cancel only partly; i.e. some terms do not drop out. The higher the power of $z^{y+p}$, the greater the number of terms which do not drop out. A preliminary test of convergence of the functions $P^{(\nu)}$ should be made at the beginning for values of $M, L, C, D, N$ and $A$ close to those which may be used in the given problem. In the case considered one obtains for $M=L=C=D=$ $N=1, A=3$ after the necessary calculations are performed:

\begin{tabular}{c|c|c|c|c|c}
\hline \hline$z^{*}$ & 0 & 0.1 & 0.2 & 0.3 & 0.6 \\
\hline$P^{\left(I_{10}\right)}$ & 1 & 0.995000 & 0.980100 & 0.955300 & 0.825300 \\
\hline$P^{\left(I_{11}\right)}$ & 0 & -0.029640 & -0.116540 & -0.257000 & -0.946860 \\
\hline$P^{\left(I_{12}\right)}$ & 0 & 0.100698 & 0.239742 & 0.367548 & 0.987782 \\
\hline
\end{tabular}

As one can see, the convergence is fairly good for small values of $z^{*}$. 
3. Partial differential equations of loaded plates with small deflections. The partial differential equation solved by Bergman [1] is homogeneous, i.e., all forms contain the function $U$. On the other hand the partial differential equations of loaded plates with small deflections are mostly non-homogeneous, i.e., they contain a term without the function $U$. In these cases one has to perform the transformation of a non-homogeneous equation into a homogeneous one, a task which is not always possible with the use of mathematical methods available today. Let us consider several cases [3]:

(a) laterally loaded isotropic plate:

$$
\frac{\partial^{4} w}{\partial x^{4}}+2 \frac{\partial^{4} w}{\partial x^{2} \partial y^{2}}+\frac{\partial^{4} w}{\partial y^{4}}=\frac{q}{D}
$$

where

$q$, intensity of a continuously distributed load,

$w$, deflection,

$$
D=\frac{E h^{3}}{12\left(1-\nu^{2}\right)}
$$

$E$, Young's modulus,

$h$, thickness of the plate,

$\nu$, Poisson's ratio.

(b) laterally loaded anisotropic plate:

$$
\begin{gathered}
D_{x} \frac{\partial^{4} w}{\partial x^{4}}+2 H \frac{\partial^{4} w}{\partial x^{2} \partial y^{2}}+D_{y} \frac{\partial^{4} w}{\partial y^{4}}=q, \\
D_{x}=\frac{E_{x}^{\prime} h^{3}}{12}, \quad D_{y}=\frac{E_{y}^{\prime} h^{3}}{12}, \quad D_{1}=\frac{E^{\prime \prime} h^{3}}{12}, \quad D_{x y}=\frac{G h^{3}}{12}, \\
H=D_{1}+2 D_{x y} .
\end{gathered}
$$

(c) isotropic plate bent by moments distributed along the edges:

$$
\frac{\partial^{4} w}{\partial x^{4}}+2 \frac{\partial^{4} w}{\partial x^{2} \partial y^{2}}+\frac{\partial^{4} w}{\partial y^{4}}=0 ;
$$

(d) isotropic plate on an elastic foundation:

$$
\frac{\partial^{4} w}{\partial x^{4}}+2 \frac{\partial^{4} w}{\partial x^{2} \partial y^{2}}+\frac{\partial^{4} w}{\partial y^{4}}=\frac{q-k w}{D}, \quad \text { where }
$$

$k=$ modulus of the foundation (pound per square inch per inch of deflection).

(e) isotropic plate bent under the combined action of lateral loads and forces in the middle plane of the plate:

$$
\frac{\partial^{4} w}{\partial x^{4}}+2 \frac{\partial^{4} w}{\partial x^{2}} \frac{\partial^{4} w}{\partial y^{2}}+\frac{1}{\partial y^{4}}=\frac{1}{D}\left[q+N_{x} \frac{\partial^{2} w}{\partial x^{2}}+N_{\nu} \frac{\partial^{2} w}{\partial y^{2}}+2 N_{x y} \frac{\partial^{2} w}{\partial x \partial y}\right]
$$

where

$N_{x}, N_{y}$, normal forces per unit length of sections of the plate perpendicular to $x$ - and $y$-directions, respectively, 
$N_{x y}$, shearing force in the direction of $y$-axis per unit length of section of the plate perpendicular to $x$-axis.

Setting $q=0$ in Eq. (38), we obtain the equation for the deflection of a plate under the action of forces in the middle plane only.

(f) isotropic plate with small initial curvature:

$$
\begin{aligned}
\frac{\partial^{4} w_{1}}{\partial x^{4}} & +2 \frac{\partial^{4} w_{1}}{\partial x^{2} \partial y^{2}}+\frac{\partial^{4} w_{1}}{\partial y^{4}}=\frac{1}{D}\left[q+N_{x} \frac{\partial^{2}\left(w_{0}+w_{1}\right)}{\partial x^{2}}\right. \\
& \left.+N_{y} \frac{\partial^{2}\left(w_{0}+w_{1}\right)}{\partial y^{2}}+2 \dot{N}_{x y} \frac{\partial^{2}\left(w_{0}+w_{1}\right)}{\partial x \partial y}\right],
\end{aligned}
$$

where $w_{0}$, initial deflection,

$w_{1}$, additional deflection.

In general we may write

$$
\Delta \Delta w+a w_{x x}+2 b w_{x y}+c w_{y y}+f w=X,
$$

where $X$, which is equal in most cases to $q / D$, may be a constant, or a function of $x$ and $y$, or sometimes is equal to zero. The general procedure is the following: one finds any particular solution of equation (40). Let it be denoted by $w_{1}$. After substituting it into (40) and subtracting this new equation from (40) one obtains the homogeneous equation,

$$
\begin{aligned}
\Delta \Delta\left(w-w_{1}\right)+a\left(w_{x x}-w_{1 x x}\right)+2 b\left(w_{x y}-\right. & \left.w_{1 x y}\right) \\
& +c\left(w_{y y}-w_{1 y y}\right)+f\left(w-w_{1}\right)=0 .
\end{aligned}
$$

Let us consider some special cases:

(a) assume $f=$ const. and $X=$ const. Put $w_{1}=$ const. $=E ; f E=X, w_{1}=X / f$,

(b) assume $f=0$, and $X, a, b, c$ are constant. Put $w_{1}=\frac{1}{2}$ const. $x^{2}, w_{1 x x}=$ const., $a \cdot$ const. $=X$, or $w_{1}=\frac{1}{2}(X / a) x^{2}$

(c) assume $a=b=c=f=0, X=$ const.; put $w_{1}=(1 / 24)$ const. $x^{4}$,

$$
\frac{\partial^{4} w_{1}}{\partial x^{4}}=\text { const. }=X, \quad \text { or } \quad w_{1}=\frac{X}{24} x^{4} \text {. }
$$

(d) assume $L(w)=X$ where $X$ is a certain polynomial in $x$ and $y$ with constant coefficients; assume also $a, b, c$, constant. Thus $X=\sum a_{\nu \mu} x^{\nu} y^{\mu}$ or $X=\sum a_{\nu \mu} \cos$ $(\nu x) \cos (\mu y)$. Let $w_{1}$ be a polynomial in $x$ and $y$ of the same degree as $X$. Thus $w_{1}=\sum A_{\nu \mu} x^{\nu} y^{\mu}$ or $w_{1}=\sum A_{\nu \mu} \cos (\nu x) \cos (\mu y)$. Substitution of $w_{1}$ and subsequent comparison of the coefficients on both sides yield the values of $A_{\nu \mu}$ in terms of $a_{\nu \mu}$. In case no such combinations like those given above can be applied, or when the coefficients $a, b, c, f, X$, are not all constant, one must. refer to the general theory of partial differential equations in order to find any particular solution or any fundamental integral. The application of Green's method may be successful in some cases. The particular case $\Delta \Delta w=f$ as well as the method of finding the fundamental integral in the case of an arbitrary linear partial differential equation with constant coefficients are treated in Reference 4 . When the equation is homogeneous, the method of linear differential operators explained above can be applied and the deflection of the plate presented in the form (20). 
4. Illustration of the method. Calculation of the Critical Stresses. (a) Formulation of the problem and explanation of the procedure. As an illustration, this method will be applied to the problem of calculating the critical stresses in an isotropic triangular plate subject to a pure uniform compression parallel to the horizontal axis. The triangle is assumed to have a right angle and both legs are equal to $l$. One of the legs coincides with the $x_{1}$-axis and the other with the $y_{1}$-axis. In this case, Eq. (38) will reduce to the form

$$
\frac{\partial^{4} w}{\partial x_{1}^{4}}+2 \frac{\partial^{4} w}{\partial x_{1}^{2} \partial y_{1}^{2}}+\frac{\partial^{4} w}{\partial y_{1}^{4}}+\tau \frac{\partial^{2} w}{\partial x_{1}^{2}}=0, \quad \text { where } \quad \tau=\frac{N_{x}}{D} .
$$

Let us transfer the figure into an $x, y$-system of coordinates such that the length of a leg equals 0.6. Keeping in mind that

$$
x=\frac{0.6 x_{1}}{l}, \quad \frac{\partial w}{\partial x_{1}}=\frac{\partial w}{\partial x} \frac{\partial x}{\partial x_{1}}=\frac{0.6}{l} \frac{\partial w}{\partial x}, \quad \frac{\partial^{2} w}{\partial x_{1}^{2}}=\left(\frac{0.6}{l}\right)^{2} \frac{\partial^{2} w}{\partial x^{2}}, \quad \text { etc. },
$$

one obtains in the $x, y$-system the following equation:

$$
\frac{\partial^{4} w}{\partial x^{4}}+2 \frac{\partial^{4} w}{\partial x^{2} \partial y^{2}}+\frac{\partial^{4} w}{\partial y^{4}}+\frac{l^{2}}{0.36} \tau \frac{\partial^{2} w}{\partial x^{2}}=0 .
$$

In case the two sides are not equal, one has to express the length of one side in terms of the length of the other, and Eq. (43) will remain the same. To each value of $N_{x}=$ const., there corresponds a set of solutions $w$ satisfying Eq. (43); but the boundary conditions need. not be satisfied. However, the boundary conditions can be written in terms of a minimal problem, and the solution $w$ so determined as to satisfy this minimal problem. This method is explained in detail below.

In order to find the first critical stress one has to find the least value from this set of $w$. To this end, we must vary $N_{x}$ and look for that value of $N_{x}$ for which the deflection $w$ reaches its minimum value. Suppose now that $\tau$ is multiplied by a number $m$. In order not to change the character of Eq. (43), we shall introduce a new system of coordinates

$$
\zeta=x m^{1 / 2}, \eta=y m^{1 / 2}, \frac{\partial w}{\partial x}=\frac{\partial w}{\partial \zeta} m^{1 / 2}, \frac{\partial^{2} w}{\partial x^{2}}=\frac{\partial^{2} w}{\partial \zeta^{2}} m, \frac{\partial^{4} w}{\partial x^{4}}=\frac{\partial^{4} w}{\partial \zeta^{4}} m^{2}, \text { etc. }
$$

Eq. (43) will be transformed into

$$
\frac{\partial^{4} w}{\partial \zeta^{4}}+2 \frac{\partial^{4} w}{\partial \zeta^{2} \partial \eta^{2}}+\frac{\partial^{4} w}{\partial \eta^{4}}+\frac{l^{2}}{0.36} \tau \frac{\partial^{2} w}{\partial \zeta^{2}}=0
$$

Thus multiplication of $\tau$ by $m$ is equivalent to the multiplication of the coordinates $x$ and $y$ by $m^{1 / 2}$ or to the introduction of new coordinates $\zeta$ and $\eta$. Assuming clamped edges all along the circumference leads to two conditions:

(i) deflection along the circumference equal to zero,

$$
\oint_{c} w d \sigma=0 \quad \text { or } \quad \oint_{c} w^{2} d \sigma=0, \quad d \sigma=\left(d \zeta^{2}+d \eta^{2}\right)^{1 / 2},
$$

(ii) the first derivative of the deflection with respect to the normal along the circumference is equal to zero, 


$$
\oint_{c} \frac{d w}{d n} d \sigma=0, \quad \text { or } \quad \oint\left(\frac{d w}{d n}\right)^{2} d \sigma=0 \text {. }
$$

If the solution is not very far from the state where (i) and (ii) are equal to zero, both conditions can be combined into

$$
L=\oint_{c} w^{2} d \sigma+\Lambda \oint_{c}\left(\frac{d w}{d n}\right)^{2} d \sigma=\min .
$$

Concerning the value of $\Lambda$, some authors (References $5,6,7$ ) assume that it is connected with the question of what ratio each of the two conditions $w=0$ and $d w / d n=$ 0 should be taken into account. If the function $w$ satisfies the boundary precisely, both $w$ and $d w / d n$ would vanish on the boundary. The particular solution chosen, however, does not satisfy the boundary conditions completely. That part of the work which arises due to the deviation from $w=0$ may be expressed as

$$
k_{1}=C_{1} \oint_{c} w^{2} d \sigma
$$

and that part of the work which arises due to the deviation from $d w / d n=0$ may be expressed as

$$
k_{2}=C_{2} \oint_{c}\left(\frac{d w}{d n}\right)^{2} d \sigma
$$

where $C_{1}$ and $C_{2}$ depend on the properties of boundary supports. The next condition imposed is the requirement that

$$
k_{1} \cong k_{2}, \quad \text { i.e., } \quad C_{1} \oint_{c} \cdot w^{2} d \sigma=C_{2} \oint_{c}\left(\frac{d w}{d n}\right)^{2} d \sigma .
$$

Therefore, the coefficient $\Lambda$ is

$$
\Lambda=C_{2} / C_{1}
$$

This requirement implies that the work of the boundary forces which arises from the chosen particular solution divides uniformly into both parts (References 5, 6). For the boundary conditions $w=0, d^{2} w / d n^{2}=0$, Bergman cites that on the basis of approximate calculations the requirement (50) is fulfilled best if one chooses $\Lambda=0.002$, 0.5 , or 200 . In the case considered in this paper, the value $\Lambda=1$ was chosen after some approximate calculations.

Equation (47) can also be written in terms of the $(x, y)$-coordinates in the following manner,

$$
\begin{gathered}
d \sigma=\left(d \zeta^{2}+d \eta^{2}\right)^{1 / 2}=\left(m d x^{2}+m d y^{2}\right)^{1 / 2}=m^{1 / 2} d s \\
L=m^{1 / 2} \oint_{c} w^{2} d s+\Lambda m^{1 / 2} \oint_{c}\left(\frac{d w}{d n}\right)^{2} d s=\min .
\end{gathered}
$$


One more condition must be superimposed on the whole system, namely, that the energy of the deflected plate should be positive. In the case considered, it is sufficient to assume that the energy

$$
E_{1}=\frac{1}{2} \iint_{0} N_{x}\left(\frac{d w}{d \zeta}\right)^{2} d \zeta d \eta>0 \quad \text { or } \quad=\text { const., }
$$

Since $N_{x}=$ const. this condition may be expressed as

$$
E=\iint_{\delta}\left(\frac{d w}{d \zeta}\right)^{2} d \zeta d \eta=m \iint_{\int}\left(\frac{d w}{m^{1 / 2} d x}\right)^{2} d x d y>0 \quad \text { or } \quad=\text { const. }
$$

It is obvious that in Eqs. (44) to (50), (53) and (54), $w$ is expressed in $\zeta, \eta$ system of coordinates. In the case considered $m=1,4,9,16,25$ and 36 was chosen.

The deflection $w$ will be expressed by $U\left(z, z^{*}\right)$ in Eq. (20). Since Eq. (42) was chosen as the starting point, only the sequences $P^{(11)}$ and $P^{(I 2)}$ will be taken into account. After all necessary calculations are completed, the imaginary part of $U\left(z, z^{*}\right)$ will be assumed to be real, since one mày easily see from Bergman's method [1] that for real partial differential equations the function $u$ in $U=u+i v$ will be real. The circumference of the triangle and its surface will be divided into several parts. In the case of a curvilinearly shaped plate, the circumference has to be transformed to a polygon and the precision of the calculation will depend on the number of sides. The coordinates $x$ and $y$ are measured for the center of each part or side and the sequences $P^{(I 1)}$ and $P^{(I 2)}$ are calculated for a finite number of terms by substitution of these measured values of $x$ and $y$. The unknown coefficients $a_{v 1}, a_{v 2}, b_{v 1}, b_{v 2}$, are determined from the minimal problem with constraints discussed previously. This leads to a set of $n$ linear equations with $n$ unknowns of the form

$$
\frac{\partial L}{\partial a_{v 1}}+\lambda \frac{\partial E}{\partial a_{v 1}}=0,
$$

where $\lambda$ is the Lagrangian multiplier. These equations will possess' solutions $a_{\nu 1}$ and $b_{\boldsymbol{p} 1}$ different from zero if and only if the determinant of the coefficients vanishes; that is, provided

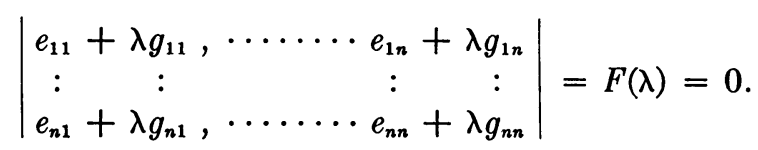

In order to find the first value of $w$ one does not need to calculate the deflection from Eq. (20). It is quite enough to take $F(\lambda)$ into account (Reference 8$)^{*}$, for consider the pair of quadratic forms

$$
\begin{aligned}
& \Phi\left(x_{1}, \cdots x_{n}\right)=\sum_{1}^{n} a_{i i} x_{i} x_{i}, \\
& \psi\left(x_{1}, \cdots x_{n}\right)=\sum_{1}^{n} b_{i i} x_{i} x_{i} .
\end{aligned}
$$

*Chapter 13, p. 169, Theorem 3. 
Then the function

$$
\Phi-\lambda \psi=\sum_{1}^{n}\left(a_{i j}-\lambda b_{i j}\right) x_{i} x_{i},
$$

has as its discriminant, the determinant

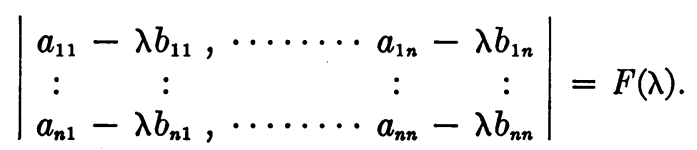

If $\lambda_{1}, \lambda_{2}, \lambda_{3} \cdots \lambda_{n}$ denote the roots of the $\lambda$-equation of the pair of forms $\Phi$ and $\psi$, $F(\lambda)=0$, then it can be proved that $\Phi$ and $\psi$ may be reduced by means of a non-singular. linear transformation to the normal forms

$$
\begin{gathered}
\lambda_{1} x_{1}^{2}+\lambda_{2} x_{2}^{2}+\cdots+\lambda_{n} x_{n}^{2}, \\
x_{1}^{2}+x_{2}^{2}+\cdots+x_{n}^{2} .
\end{gathered}
$$

Thus we obtain a pair of quadratic forms, one with çefficients $\lambda_{1}, \lambda_{2}, \lambda_{3} \cdots \lambda_{n}$, and the other with all coefficients equal to 1 . Hence we may consider the pair of quadratic forms (60) instead of (57). Since one looks for the first value, only $\lambda_{1}$ has to be taken into account for various values of $m$. The minimum of the function $\lambda_{1}=f(m)$, which may be plotted as a diagram, gives the value of $m$ for the critical stress.

(b) Calculations of the sequences $K_{n v} P^{(11 n)}\left(z^{*}\right) z^{n+\nu}$ and $K_{n v} P^{(I 2 n)}\left(z^{*}\right) z^{n+\nu}$. Let us take the first three terms of the first sequence (18):

$$
\begin{aligned}
U\left(z, z^{*}\right)_{1}=P^{(110)}\left(z^{*}\right) z^{\nu} & +\frac{1}{2} P^{(I 11)}\left(z^{*}\right) \frac{z^{\nu+1}}{(\nu+1)} \\
& +\frac{3}{4} P^{(I 12)}\left(z^{*}\right) \frac{z^{\nu+2}}{(\nu+1)(\nu+2)} .
\end{aligned}
$$

If we substitute (23), (33) etc. into (61), comparison of (2) and (43) gives:

$$
\begin{aligned}
a & =\frac{l^{2}}{0.36} \frac{N_{x}}{D}, \quad b=c=d=e=f=0, \quad M_{2}=A_{1}=A_{2}=C=0, \\
L & =\frac{a}{8}=\frac{l^{2}}{0.36} \frac{N_{x}}{8 D}, \quad M_{1}=\frac{a}{16}=\frac{l^{2}}{0.36} \frac{N_{x}}{16 D}, \\
N & =\frac{a}{16}=\frac{l^{2}}{0.36} \frac{N_{x}}{16 D}=\frac{l^{2} N_{x}}{5.76 D}, \\
A=D & =C=0 .
\end{aligned}
$$

Let us assume in (1) that $M=1, L=2, N=1$ and substitute these values into (23), (33) etc. and into (61). After necessary calculations have been made we obtain

$$
\begin{gathered}
U\left(z, z^{*}\right)_{1}=\left(\cos z^{*}\right) z^{\nu}+\left(\sin z^{*}-z^{*} \cos z^{*}\right) \frac{z^{\nu+1}}{(\nu+1)} \\
+\frac{1}{2} z^{*^{2}}\left(\cos z^{*}\right) \frac{z^{\nu+2}}{(\nu+2)(\nu+1)}
\end{gathered}
$$


Let us assume $\nu=0,1,2,3$, and decompose (63) into the real and the imaginary parts, calling the real part $\psi_{v 1} ;$ and the imaginary $\psi_{\nu 2}$. Then

$$
\begin{aligned}
\psi_{01}=x \sin x \cosh y+y \cos x \sinh y & \\
+ & {\left[\frac{x^{4}+y^{4}}{4}+\frac{x^{2} y^{2}}{2}-x^{2}-y^{2}+1\right] \cos x \cosh y, } \\
\psi_{02}=-i x & \cos x \sinh y+i y \sin x \cosh y \\
+ & {\left[\frac{x^{4}+y^{4}}{4}+\frac{x^{2} y^{2}}{2}-x^{2}-y^{2}+1\right] i \sin x \sinh y . }
\end{aligned}
$$

It can be easily seen that $\psi_{v 2}$ can be obtained from $\psi_{v 1}$ changing $(\sin x)$ into $(-i \cos x)$, $(\cos x)$ into $(i \sin x),(\sinh y)$ into $\cosh y)$ and $(\cosh y)$ into $(\sinh y)$. Consequently, it is enough to give the results for $\psi_{v 1}$ and $\Phi_{v 1}$ only.

$$
\begin{aligned}
& \psi_{11}=\left[\frac{x^{5}}{12}+\frac{x^{3} y^{2}}{6}+\frac{y^{4} x}{12}-\frac{x^{3}}{2}-\frac{x y^{2}}{2}+x\right] \cos x \cosh y \\
& -\left[\frac{x^{4} y}{12}+\frac{x^{2} y^{3}}{6}+\frac{y^{5}}{12}-\frac{y^{3}}{2}-\frac{x^{2} y}{2}+y\right] \sin x \sinh y \\
& +\left[\frac{x^{2}}{2}-\frac{y^{2}}{2}\right] \sin x \cosh y+x y \cos x \sinh y, \\
& \psi_{21}=\left[\frac{x^{6}+x^{4} y^{2}-x^{2} y^{4}-y^{6}}{24}+\frac{x^{4}-y^{4}}{3}+x^{2}-y^{2}\right] \cos x \cosh y \\
& -\left[\frac{x^{5} y+x y^{5}}{12}+\frac{x^{3} y^{3}}{6}+\frac{2 x^{3} y+2 x y^{3}}{3}+2 x y\right] \sin x \sinh y \\
& +\frac{x^{3}-3 x y^{2}}{3} \sin x \cosh y+\frac{3 x^{2} y-y^{3}}{3} \cos x \sinh y, \\
& \psi_{31}=\left[\frac{x^{7}-x^{5} y^{2}-5 x^{3} y^{4}-3 x y^{6}}{40}-\frac{x^{5}-2 x^{3} y^{2}-3 x y^{4}}{4}+x^{3}-3 x y^{2}\right] \cos x \cosh y \\
& -\left[\frac{3 x^{6} y+5 x^{4} y^{3}+x^{2} y^{5}-y^{7}}{40}\right. \\
& \left.-\frac{3 x^{4} y+2 x^{2} y^{3}-y^{5}}{4}+3 x^{2} y-y^{3}\right] \sin x \sinh y \\
& +\frac{x^{4}-6 x^{2} y^{2}+y^{4}}{4} \sin x \cosh y+\left(x^{3} y-x y^{3}\right) \cos x \sinh y \text {. }
\end{aligned}
$$


In the same way the function $U\left(z, z^{*}\right)_{2}$ for $P^{(I 2)}$ can be calculated; namely,

$$
\begin{aligned}
U\left(z, z^{*}\right)_{2}= & P^{(120)}\left(z^{*}\right) z^{\nu}+\frac{1}{2} P^{(I 21)}\left(z^{*}\right) \frac{z^{\nu+1}}{(\nu+1)}+\frac{3}{4} P^{(I 12)}\left(z^{*}\right) \frac{z^{\nu+2}}{(\nu+1)(\nu+2)} \\
= & \left(\sin z^{*}\right) z^{\nu}-z^{*}\left(\sin z^{*}\right) \frac{z^{\nu+1}}{(\nu+1)} \\
& +\left(\sin z^{*}-z^{*} \cos z^{*}+\frac{1}{2} z^{*^{2}} \sin z^{*}\right) \frac{z^{\nu+2}}{(\nu+1)(\nu+2)} .
\end{aligned}
$$

Let us call the real part $\Phi_{v 1}$, and the imaginary $\Phi_{v 2}$. Then

$$
\begin{gathered}
\Phi_{01}=\left[\frac{x^{4}+2 x^{2} y^{2}+y^{4}}{4}-\frac{x^{2}+3 y^{2}}{2}+1\right] \sin x \cosh y \\
+x y \cos x \sinh y-\frac{x^{3}+x y^{2}}{2} \cos x \cosh y+\frac{x^{2} y+y^{3}}{2} \sin x \sinh y \\
\Phi_{11}=\left[\frac{x^{5}+2 x^{3} y^{2}+x y^{4}}{12}-\frac{x^{3}+3 x y^{2}}{3}+x\right] \sin x \cosh y \\
+\left[\frac{x^{4} y+2 x^{2} y^{3}+y^{5}}{12}-\frac{2 y^{3}}{3}+y\right] \cos x \sinh y \\
-\frac{x^{4}-y^{4}}{6} \cos x \cosh y+\frac{x^{3} y+x y^{3}}{3} \sin x \sinh y
\end{gathered}
$$

$-\frac{x^{5}-2 x^{3} y^{2}-3 x y^{4}}{12} \cos x \cosh y+\frac{3 x^{4} y+2 x^{2} y^{3}-y^{5}}{12} \sin x \sinh y$

$$
\begin{aligned}
\Phi_{31}=\left[\frac{x^{7}-x^{5} y^{2}-5 x^{3} y^{4}-3 x y^{6}}{40}+\frac{15 x y^{4}-x^{5}-10 x^{3} y^{2}}{30}+x^{3}-3 x y^{2}\right] \sin x \cosh y \\
+\left[\frac{3 x^{6} y+5 x^{4} y^{3}+x^{2} y^{5}-y^{7}}{40}\right. \\
\left.+\frac{2 y^{5}-10 x^{2} y^{3}}{30}+3 x^{2} y-y^{3}\right] \cos x \sinh y \\
-\left[\frac{x^{6}-5 x^{4} y^{2}-5 x^{2} y^{4}+y^{6}}{20}\right] \cos x \cosh y+\frac{x^{5} y-x y^{5}}{5} \sin x \sinh y
\end{aligned}
$$


(c) Graphical representation of the particular solutions. A graphical representation for the particular solutions can be obtained in the following manner:

$$
\text { Let } \begin{aligned}
z & =r e^{i \phi}, & z^{\nu} & =r^{\nu}(\cos \nu \phi+i \sin \nu \phi), \\
z^{*} & =r e^{-i \phi}, & z^{* \nu} & =r^{\nu}(\cos \nu \phi-i \sin \nu \phi) .
\end{aligned}
$$

The values of $\psi_{v n}$ and $\Phi_{v n}$ can be calculated for some number of points, say for $r=0.2$, $0.4,0.6,0.8,1.0$ and $\phi=15^{\circ}, 30^{\circ}, 45^{\circ} \cdots 90^{\circ}$. These points are plotted on a polar diagram $r, \phi$ separately for $\psi_{\nu 1}$ and $\Phi_{\nu 2}$. Points of the equal value of $\psi_{\nu n}$ and $\Phi_{\nu n}$ can be obtained by interpolation and the lines connecting these points will give the graphical representation of the particular solutions $\psi_{v n}$ and $\Phi_{p n}$. Some of the results obtained in this way are shown in Fig. 1. Similar graphs can be obtained for $P^{\left(I I_{n}\right)}$. The real part of $P^{(I n)}$ will be the same as that of $P^{(I I n)}$ and the imaginary part of $P^{(I I n)}$ will have the same magnitude but the opposite sign to that of $P^{(I n)}$.

(d) Calculation of derivatives. The derivative $\dot{d} w / d n$ has to be calculated for Eq. (52):

$$
\frac{d w}{d n}=\frac{d \psi_{\nu n}}{d n}=\frac{\partial \psi_{\nu n}}{\partial x} \frac{\partial x}{\partial n}+\frac{\partial \psi_{\nu n}}{\partial y} \frac{\partial y}{\partial n}=\frac{1}{2^{1 / 2}}\left[\frac{\partial \psi_{\nu n}}{\partial x}+\frac{\partial \psi_{v n}}{\partial y}\right],
$$

since $\partial x / \partial n=\cos \alpha=1 \% 2^{1 / 2}, \partial y / \partial n=\sin \alpha=1 / 2^{1 / 2}, \alpha=45^{\circ}$.

For the horizontal and the vertical sides Eq. (74) changes to $d \psi_{\nu n} / d n=\left(d \psi_{\nu n} / d y\right)_{\nu=0}$ and $d \psi_{v n} / d n=\left(d \psi_{v n} / d x\right)_{x=0}$ respectively. For Eq. (54) $d \psi_{v n} / d x,\left(d \psi_{v n} / d x\right)_{x=0}$ and $\left(d \psi_{\nu n} / d x\right)_{y=0}$ must be calculated. The results are given below:

$$
\begin{aligned}
\frac{\partial \psi_{01}}{\partial x}=\left[x^{3}\right. & \left.+x y^{2}-x\right] \cos x \cosh y-\left[\frac{x^{4}+y^{4}}{4}+\frac{x^{2} y^{2}}{2}-x^{2}-y^{2}\right] \sin x \cosh y \\
& -y \sin x \sinh y,
\end{aligned}
$$

$$
\begin{aligned}
& \frac{\partial \psi_{01}}{\partial y}=\left[x^{2} y+y^{3}-y\right] \cos x \cosh y+\left[\frac{x^{4}+y^{4}}{4}+\frac{x^{2} y^{2}}{2}-x^{2}-y^{2}+2\right] \cos x \sinh y \\
& +x \sin x \sinh y \\
& \frac{d \psi_{01}}{d n}=\frac{1}{2^{1 / 2}}\left[\left(x^{3}+x y^{2}-x+x^{2} y+y^{3}-y\right) \cos x \cosh y\right. \\
& -\left(\frac{x^{4}+y^{4}}{4}+\frac{x^{2} y^{2}}{2}-x^{2}-y^{2}\right) \sin x \cosh y \\
& +\left(\frac{x^{4}+y^{4}}{4}+\frac{x^{2} y^{2}}{2}-x^{2}-y^{2}+2\right) \cos x \sinh y \\
& +(x-y) \sin x \sinh y]
\end{aligned}
$$

To obtain the derivatives of $\psi_{02}$ the same changes as explained above have to be introduced. In a similar way, all the necessary derivatives $\partial \psi_{11} / \partial x, \partial \psi_{11} / \partial y$, etc., $\partial \phi_{01} / \partial x$, $\partial \phi_{01} / \partial y$, etc. were calculated. 

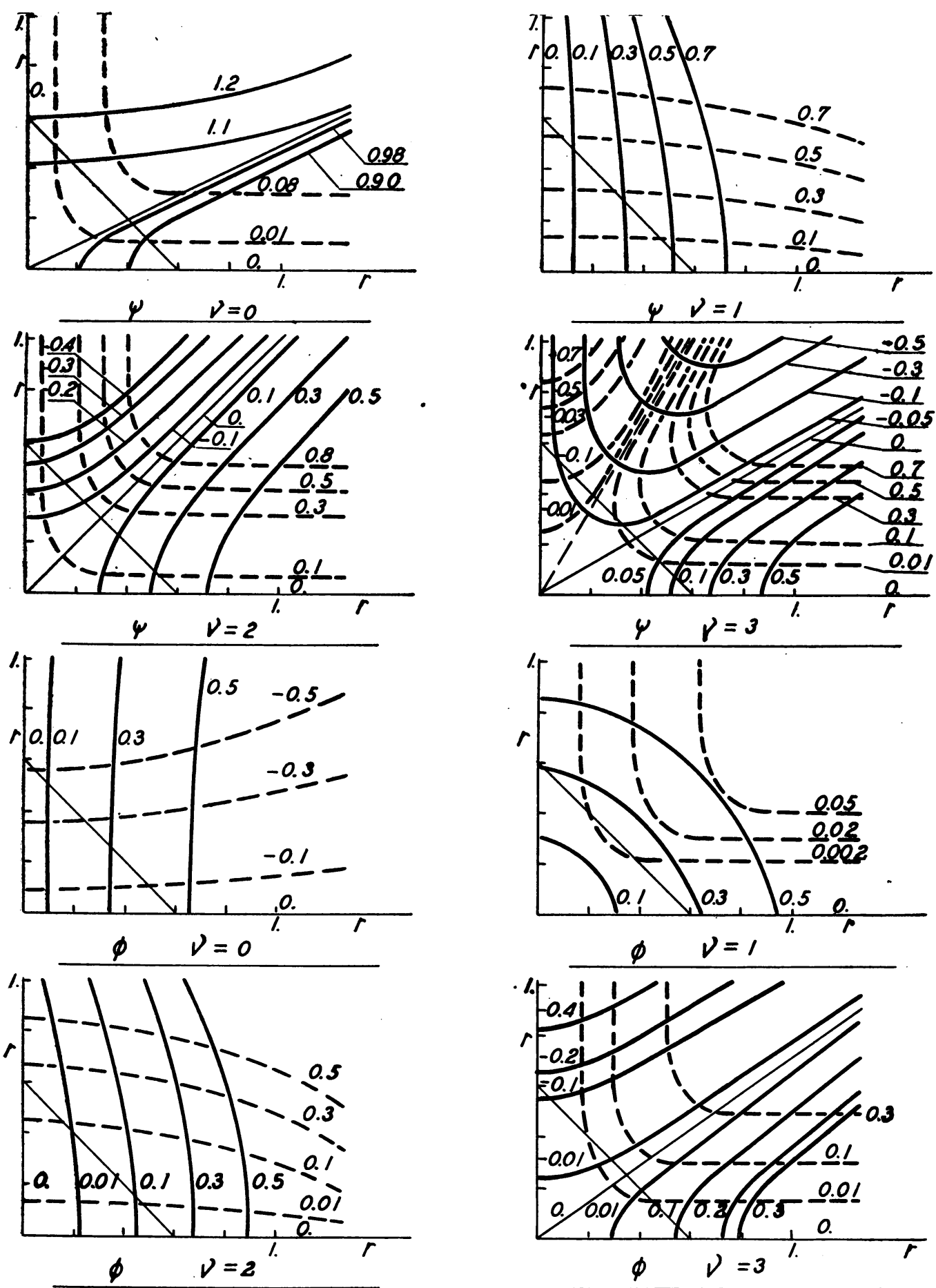

Fig. 1. The functions of $\psi$ and $\phi$ in polar coordinates $(r, \varphi)$. 
(e) Procedure for calculation-It is easily seen that the number of operations involved in the evaluation the above equations may reach a hundred thousand or more. For this reason punch-card-machines were used. The circumference and the surface of the triangle were divided into some number of parts; for each part, the coordinates of the center point were defined and substituted into the above equations for $\psi_{v n}, \Phi_{v_{n}}$ and for all necessary derivatives.

The calculations were performed simultaneously for six values of $m$, which is equivalent to the multiplication of all terms in $x$ and $y$ of the first degree by $m^{1 / 2}$ of the second degree by $m$, of the fourth degree by $m^{2}$, etc. Of course, the values of the trigonometric and hyperbolic functions also have to be changed with change in $m$. In the next step, the squares in (52) and (53) were calculated. This is equivalent to the squaring of 16 term polynomials, since 4 groups $\psi_{\nu 1}, \psi_{\nu 2}, \Phi_{\nu 1}, \Phi_{\nu 2}$ with 4 terms in each group weré taken into account. The integrations in Eq. (52) and (53) were replaced by multiplication of each $\psi_{\nu n}$ by the corresponding partial length or surface and summation on the punch-card-machines. Next, the smallest roots of (56) were found in several cases. In order to obtain a good picture of the influence of the order of the determinant on the value of $\lambda$, the value of the smallest root, $\lambda$, was calculated for determinants of various orders. Determinants were calculated for all six values of $m$. The results of these calculations are given in Table I for three values of $m$ in the neighborhood of the minimum. All determinants were calculated by the use of punch-card-machines.

TABLE 1. $\lambda=f(m)$

\begin{tabular}{c|c|c|c|c|c}
\hline \hline & & & \multicolumn{2}{c}{ Value of } \\
No. & $\begin{array}{c}\text { Coefficients } \\
\text { Used }\end{array}$ & $\begin{array}{c}\text { Order of } \\
\text { determ. }\end{array}$ & $m=3^{2}$ & $m=4^{2}$ & $m=5^{2}$ \\
\hline 1 & $a_{01}$ & 1 & -54.5 & -5.601058 & -8.660602 \\
\hline 2 & $a_{01}, b_{01}$ & 2 & -2.8 & -0.15674675 & -0.962 \\
\hline 3 & $a_{01}, a_{02}$ & 2 & -8.77342465 & -0.163899924 & -1.203058734 \\
\hline 4 & $a_{01}, a_{02}$, & 4 & -0.0394278 & -0.000211601 & -0.603463788 \\
\hline$b_{01}, b_{02}$ & & & \\
\hline
\end{tabular}

To obtain more precise value of $\lambda_{c r}$, the values of the two functions $\psi_{01}, \psi_{02}$ were calculated for all integers between 17 and 24 . Table 1 shows that it is sufficient to take into account only the first function $\psi_{01}$ for the location of $\lambda_{c r}$. The results of calculations for $\psi_{01}$ are given below.

\begin{tabular}{lcccc}
\multicolumn{5}{c}{ TABLE $2 . \lambda=f(m)$} \\
$m$ & 17 & 18 & 19 & 20 \\
$\lambda$ & -5.344245 & -5.288552 & -5.225255 & -5.178953 \\
$m$ & 21 & 22 & 23 & 24 \\
$\lambda$ & -5.306672 & -5.329772 & -5.639540 & -6.576221
\end{tabular}

Thus $\lambda_{c r}$ is at $m=20$. To obtain still more precise value of $\lambda_{c r}$ one may repeat the calculations for several decimal values between 19 and 21 . 
The value of the critical stress is given by Eq. (62):

$$
\begin{gathered}
m N=\frac{m l^{2} N_{x}}{5.76 D}, \quad\left(m N_{x}\right)=N_{x c r}, \quad N=1, \\
N_{x c r}=20 \times 5.76 \frac{D}{l^{2}}=115.2 \frac{D}{l^{2}} .
\end{gathered}
$$

This may be considered as the first approximation* of $N_{x c r}$.

This method may also be applied to the case where the coefficients in Eq. (40) are analytic functions in $x$ and $y$. Since the procedure in this case remains the same as in the case of a partial differential equation of elliptic type and second order one may refer to Reference 10.

The whole procedure may be performed by a graphical method. Diagrams for the particular solutions, for the first and second derivatives, can be plotted as explained above, and the corresponding values read off for each plate shape considered. There is a possibility of preparing a set of such diagrams for various values of forces $N_{x}, N_{y}$, $N_{x y}$, taken in certain intervals, similar to charts used in practice for buckling of columns. This technique has been used in order to check the results obtained on the punch-cardmachines; the agreement was bound to be astonishingly good.

Similarly, in the case where the coefficients $M, L, N \cdots$ in (1) are analytic functions of $z$ and $z^{*}$, one must refer to the general method of solution of such equations by use of punch-card-machines [10].

\section{REFERENCES}

1. Stefan Bergman, Solutions of linear partial differential equations of the fourth order, Duke Mathematical Journal 11, 617-649 (1944).

2. E. L. Ince, Ordinary differential equations, Dover Publications, New York, 1944, p. 122.

3. A. Nadai, Die elastischen Platten, J. Springer, Berlin, 1925.

4. P. Frank and R. v. Mises, Die Differential- und Integralgleichungen der Mechanik und Physik, 2-d edition, M. S. Rosenberg, New York, 1943.

5. Stefan Bergmän, Neuere Probleme aus der Flugzeugstatik. Über die Knickung von rechteckigen Platten bei Schubbeanspruchung, Prikl. Mat. Mekh. 2, 207-224 (1935).

6. Stefan Bergman, Über Schubknickung von isotropen and aniostropen Platten, Proc. 3-d Internat. Congr. App. Mech., Stockholm, 1930, pp. 82-87.

7. H. Reissner, and S. Bergman, Neuere Probleme aus der Flugzeugstatik, Z. Flugtech. Motorluftschiffahrt 23, 6-12 (1932).

8. Maxime Bocher, Introduction to higher algebra, The Macmillan Company, New York, 1929.

9. Thornton C. Fry, Some numerical methods for locating roots of polynomials, Q. Appl. Math. 3, 89-105 (1945).

10. Stefan Bergman, Construction of a complete set of solutions of a linear partial differential equation in two variables by use of punch-card-machines, Q. Appl. Math. 4, 233-245 (1946).

*For comparison purposes several values are given below: square plate, built-in edges, compression in $x$-direction, $N_{x c r}=105\left(D / a^{2}\right)$; square plate, simply supported edges, compression in $x$-direction, $N_{x c r}=4 \pi^{2}\left(D / a^{2}\right)$; equilateral triangular plate, simply supported edges, uniformly compressed, $N_{c r}=$ $4 \pi^{2}\left(D / a^{2}\right), a=$ height. 